Gut and Liver, Vol. 9, No. 4, July 2015, pp. 433-434

\title{
Is Lipase Supplementation before a High Fat Meal Helpful to Patients with Functional Dyspepsia?
}

\author{
Seon-Young Park and Jong-Sun Rew \\ Division of Gastroenterology and Hepatology, Department of Internal Medicine, Chonnam National University Medical School, Gwangju, Korea
}

See "Lipase Supplementation before a High-Fat Meal Reduces Perceptions of Fullness in Healthy Subjects" by Max E. Levine, et al. on page 464, Vol. 9. No. 4, 2015

Functional dyspepsia (FD) is a highly prevalent disorder, characterized by persistent or recurrent pain or discomfort centered in the upper abdomen without evidence of organic that might explain the symptoms. Several factors have been proposed to play a role: delayed gastric emptying, Helicobacter pylori infection, hypersensitivity to gastric distension, impaired gastric accommodation to a meal, altered duodenal sensitivity to lipids or acids, abnormal duodenojejunal motility, and central nervous system. ${ }^{1}$ However, none of these abnormalities are able to completely account for the dyspepsia symptom complex. Epidemiologic studies, both in the United States and Europe, have shown that $50 \%$ to $80 \%$ of subjects with FD indicate that their symptoms are meal-related. ${ }^{2}$ Meal digestion is associated with diverse change in the gastrointestinal function and potential pathophysiologic mechanism. After ingestion of meal, patient with FD experience a marked rise in the intensity of their symptoms such as epigastric burning, epigastric pain, fullness, bloating, nausea and belching. Many patients report that ingestion of food, particularly meals containing fat, induces, or exacerbates, their symptoms. Many patients complain that ingestion of food, particularly meals containing fat, induces, or exacerbates, their symptoms. Previous study showed that in FD patients, a high fat meal induced more symptoms such as nausea and pain than an isocaloric high carbohydrate meal. ${ }^{3}$ They suggested that the effects of the meals were related to the gut hormones involved in appetite regulation. Fasting and postprandial plasma cholecystokinin (CCK) concentration were greater and peptide-YY concentrations were lower in FD and in healthy controls. And, they showed that significant correlations between the scores for nausea and pain and plasma CCK concentrations. ${ }^{3}$ In Levine's study, ${ }^{4}$ stomach fullness, bloating, and nausea increased significantly 10 minutes after ingestion of fatty meal in 16 healthy volunteers. Levine et al. ${ }^{4}$ also analyzed the effect of fatty meal on gastric myoelectrical activity. After ingestion of fatty meal, normal gastric myoelectrical activity decreased and tachygastria increased in healthy volunteers. ${ }^{4}$ Previous study showed similar findings in patients with functional dyspepsia. ${ }^{5}$ Patients with FD revealed a preprandial increase in tachygastria compared to controls. And also, FD patients with delayed gastric emptying showed pre- and postprandial tachygastria than FD patients with normal gastric emptying. ${ }^{5}$ In a Polish study, FD patients showed dysrhythmia, especially bradygastria on electrogastrography (EGG). And also, the EGG records were different in the two forms of dyspepsia: in patients with postprandial distress syndrome there was statistically significant larger number of tachygastria than in the patients with epigastric pain syndrome. ${ }^{6}$

FD is a clinical problem of considerable magnitude for the health care system due to its high prevalence and the chronic and recurrent nature of symptoms. However, the therapeutic options for a clinician are limited and far from optimal: pharmacological therapies often fail. Levine et al. ${ }^{4}$ showed that high fat meal induced the similar symptoms in FD patients. Therefore, they speculated that lipase supplementation with meals might provide relief of symptoms. Previous study showed that pancrealipase reduced postprandial symptoms such as bloating, gas and fullness. ${ }^{7}$ However, in that study, there was no significant effect on gas production which was expressed by breath $\mathrm{H}_{2}$ and $\mathrm{CH}_{4}$ concentration. Levine's study ${ }^{4}$ also showed that lipase supplementation significantly reduced stomach fullness without change of EGG. They also did not show the physiological mech-

Correspondence to: Seon-Young Park

Department of Internal Medicine, Chonnam National University Hospital, Chonnam National University Medical School, 42 Jebong-ro, Dong-gu, Gwangju 501-757, Korea

Tel: +82-62-220-6296, Fax: +82-62-228-1330, E-mail: drpsy@naver.com pISSN 1976-2283 eISSN 2005-1212 http://dx.doi.org/10.5009/gnl15206

(a) This is an Open Access article distributed under the terms of the Creative Commons Attribution Non-Commercial License (http://creativecommons.org/licenses/by-nc/4.0) which permits unrestricted non-commercial use, distribution, and reproduction in any medium, provided the original work is properly cited. 
anism to explain the decreased fullness after lipase supplement. Ingestion of fatty meal was associated with release of enteric hormones and dysrhythmia of stomach (tachygastria), which may contribute to delayed gastric emptying. Furthermore, lipase supplementation may be helpful in control of FD symptom such as postprandial symptoms. However, until now, small size and short-term monitoring of the studies are insufficient to draw any conclusions for effect of fatty meal on symptoms of FD, their pathomechanisms and pharmachologic therapy. We expect large-scale study for elucidating the mechanism to explain the effect of lipase supplementation before high fat meal in FD patients.

\section{CONFLICTS OF INTEREST}

No potential conflict of interest relevant to this article was reported.

\section{REFERENCES}

1. Pen J. Diet in the etiology and management of functional dyspepsia.
In: Shaffer EA, Curley MA, eds. Dyspepsia: advances in understanding and management. Rijeka: InTech, 2013:95-109.

2. Feinle-Bisset C, Vozzo R, Horowitz M, Talley NJ. Diet, food intake, and disturbed physiology in the pathogenesis of symptoms in functional dyspepsia. Am J Gastroenterol 2004;99:170-181.

3. Pilichiewicz AN, Feltrin KL, Horowitz M, et al. Functional dyspepsia is associated with a greater symptomatic response to fat but not carbohydrate, increased fasting and postprandial CCK, and diminished PYY. Am J Gastroenterol 2008;103:2613-2623.

4. Levine ME, Koch SY, Koch KL. Lipase supplementation before a high-fat meal reduces perceptions of fullness in healthy subjects. Gut Liver 2015;9:464-469.

5. Pfaffenbach B, Adamek RJ, Bartholomäus C, Wegener M. Gastric dysrhythmias and delayed gastric emptying in patients with functional dyspepsia. Dig Dis Sci 1997;42:2094-2099.

6. Foryś S, Rudnicki C, Walecka-Kapica E, Chojnacki J. Electrogastrography in various clinical forms of functional dyspepsia. Pol Merkur Lekarski 2009;26:373-377.

7. Suarez F, Levitt MD, Adshead J, Barkin JS. Pancreatic supplements reduce symptomatic response of healthy subjects to a high fat meal. Dig Dis Sci 1999;44:1317-1321. 